EPJ Web of Conferences 57, 01002 (2013)

DOI: $10.1051 /$ epjconf/20135701002

(C) Owned by the authors, published by EDP Sciences, 2013

\title{
Strongly interacting Fermi gases
}

\author{
W. Bakr, L.W. Cheuk, M.J.-H. Ku, J.W. Park, A.T. Sommer, S. Will, C.-H. Wu, \\ T. Yefsah and M.W. Zwierlein ${ }^{\mathrm{a}}$
}

Department of Physics, MIT-Harvard Center for Ultracold Atoms, and Research Laboratory of
Electronics, MIT, Cambridge, Massachusetts 02139, USA

\begin{abstract}
Strongly interacting gases of ultracold fermions have become an amazingly rich test-bed for many-body theories of fermionic matter. Here we present our recent experiments on these systems. Firstly, we discuss high-precision measurements on the thermodynamics of a strongly interacting Fermi gas across the superfluid transition. The onset of superfluidity is directly observed in the compressibility, the chemical potential, the entropy, and the heat capacity. Our measurements provide benchmarks for current many-body theories on strongly interacting fermions. Secondly, we have studied the evolution of fermion pairing from three to two dimensions in these gases, relating to the physics of layered superconductors. In the presence of p-wave interactions, Fermi gases are predicted to display toplogical superfluidity carrying Majorana edge states. Two possible avenues in this direction are discussed, our creation and direct observation of spin-orbit coupling in Fermi gases and the creation of fermionic molecules of ${ }^{23} \mathrm{Na}^{40} \mathrm{~K}$ that will feature strong dipolar interactions in their absolute ground state.
\end{abstract}

\section{INTRODUCTION}

Strongly interacting Fermi gases are ubiquitous in Nature. We find them as electron gases in modern materials, such as high-temperature superconductors or colossal magneto resistance materials, as neutrons in neutron stars, or as quarks in the quark-gluon plasma of the Early Universe. Ultracold gases of fermionic atoms near Feshbach resonances realize a unique form of fermionic matter where interactions between fermions can be tuned at will. This allowed the observation of pairing and superfluidity in the crossover between Bardeen-Cooper-Schrieffer (BCS) superfluidity of long-range pairs and Bose-Einstein condensation (BEC) of tightly bound molecules [1-3].

While fermionic superfluidity was directly demonstrated throughout the BEC-BCS crossover via the observation of quantized vortices [4], without any precise knowledge of the equation of state the field lacked reliable thermometry. In recent years, the thermodynamics of this novel form of fermionic matter has been a focus of great attention [5-11]. We have recently developed a new technique, discussed in section 2 below, that allows to determine the homogeneous (i.e. not trap-averaged) equation of state of any gas directly from the density profile [11]. This allowed the direct observation of superfluidity in the thermodynamics of the unitary Fermi gas, where interactions are as strong as allowed by quantum mechanics.

To enhance the role of interactions even further, one can confine the paired gas in optical lattices. This allowed us to study the evolution of fermion pairing from three to two dimensions, see section 3 , and confirm that pairing is strongly enhanced in lower dimensions [12].

Going beyond s-wave pairing might allow the realization of topological superfluidity with cold atoms that carry Majorana edge states, relevant for quantum computation [13]. Our group is pursuing

\footnotetext{
ae-mail: zwierlein@mit.edu
}

This is an Open Access article distributed under the terms of the Creative Commons Attribution License 2.0, which permits unrestricted use, distribution, and reproduction in any medium, provided the original work is properly cited. 
several directions towards this goal, namely spin-orbit coupling of Fermi gases [14] (section 4) and the formation of fermionic molecules of ${ }^{23} \mathrm{Na}^{40} \mathrm{~K}$ that possess a strong dipole moment (section 5).

\section{THERMODYNAMICS OF A STRONGLY INTERACTING FERMI GAS}

Fermi gases right at the Feshbach resonance are universal, as there is no length scale set by the interaction strength [15]. The only remaining length scales are the interparticle spacing, set by the density $n$, and the de Broglie wavelength $\lambda$, set by the temperature. At low temperatures, the system's properties will be closely related to that of dilute neutron matter, where the scattering length has also much larger magnitude than the interparticle spacing. In the superfluid phase, the coherence length of the fermion pairs must necessarily be on the order of the interparticle spacing, a limit that high-temperature superconductors are closely realizing as well [16]. Theoretical studies of strongly interacting Fermi systems are difficult due to the absence of a small parameter. The unitary Fermi gas thus becomes a benchmark system that allows tests of various many-body approaches.

The entire thermodynamic information of a given system is encoded in its equation of state (EoS), the relation between its thermodynamic variables. A precision measurement of the EoS will also reveal the location of phase transitions, the nature of phases (e.g. whether the normal phase of strongly interacting Fermi gases contains preformed pairs or whether it is a Fermi liquid) and the ground state energy.

In our work, we directly obtain the compressibility, density and pressure of the gas, and show that these three thermodynamic quantities directly yield the full equation of state of the unitary Fermi gas [11]. We directly observe the superfluid phase transition in the compressibility, the chemical potential, the entropy, and the heat capacity. Our measurements provide benchmarks to current manybody theories of strongly interacting fermionic matter.

The initial step in obtaining the equation of state is to obtain an accurate measurement of the density $n$ as a function of the local potential $V$. For this, the column density in our cylindrically symmetric atom trap is inverted via the inverse Abel transform to yield the 3D density [17]. The potential along the axial direction is harmonic, given by a magnetic field curvature, and is precisely known. Using the fact that equidensity lines correspond to equipotential lines one obtains the equipotential contours also along the radial direction. Averaging along these equipotential lines results in increased signal to noise in the density $n$ as a function of the potential $V$.

Knowing $n(V)$, we can obtain the local pressure at each potential value $V$ by a simple integral: $P(V)=\int_{V}^{\infty} \mathrm{d} V^{\prime} n\left(V^{\prime}\right)$. We find one more thermodynamic variable by taking the derivative of $n(V)$, the change of the density with respect to the potential, which yields the compressibility: $\kappa(V)=$ $-n^{-2} \mathrm{~d} n / \mathrm{d} V$. The density, pressure and compressibility together give the compressibility equation of state $\kappa(n, P)$. Note that our method is general, it is not restricted to the unitary Fermi gas, and it does not rely on any fit or external thermometer, as in $[9,10]$.

For the unitary Fermi gas, the absence of an interaction-dependent length scale dictates that all thermodynamic quantities can be expressed by those for a non-interacting Fermi gas at zero temperature, times universal functions of $n \lambda^{3}$. We thus define the normalized compressibility $\tilde{\kappa} \equiv \kappa / \kappa_{0}$ and the normalized pressure $\tilde{p} \equiv P / P_{0}$, where $\kappa_{0}=3 / 2\left(n E_{F}\right)^{-1}$ and $P_{0}=2 / 5 n E_{F}$ are the compressibility and pressure at same density for a non-interacting Fermi gas at $T=0$, with $E_{F}=\hbar^{2}\left(3 \pi^{2} n\right)^{2 / 3} /(2 m)$ the Fermi energy. Thanks to universality at the Feshbach resonance, any measurement on an atomic cloud at any temperature, atom number, and trapping potential must produce the same universal curve $\tilde{\kappa}(\tilde{p})$. By averaging over numerous profiles, we obtain a low noise measurement of the compressibility equation of state $\tilde{\kappa}(\tilde{p})$.

The method is tested on a non-interacting Fermi gas, shown in Fig. 1(a), demonstrating good agreement with the known EoS of a non-interacting gas. Our measurement for the strongly interacting Fermi gas validates the high-temperature (high $\tilde{p}$ ) virial expansion that is known to third order [19]. A jump in the compressibility signals the normal-to-superfluid transition at around $\tilde{p}=0.55$. In the $T=0$ limit, the chemical potential is proportional to the Fermi energy, $\mu=\xi E_{F}$, with $\xi$ the Bertsch 

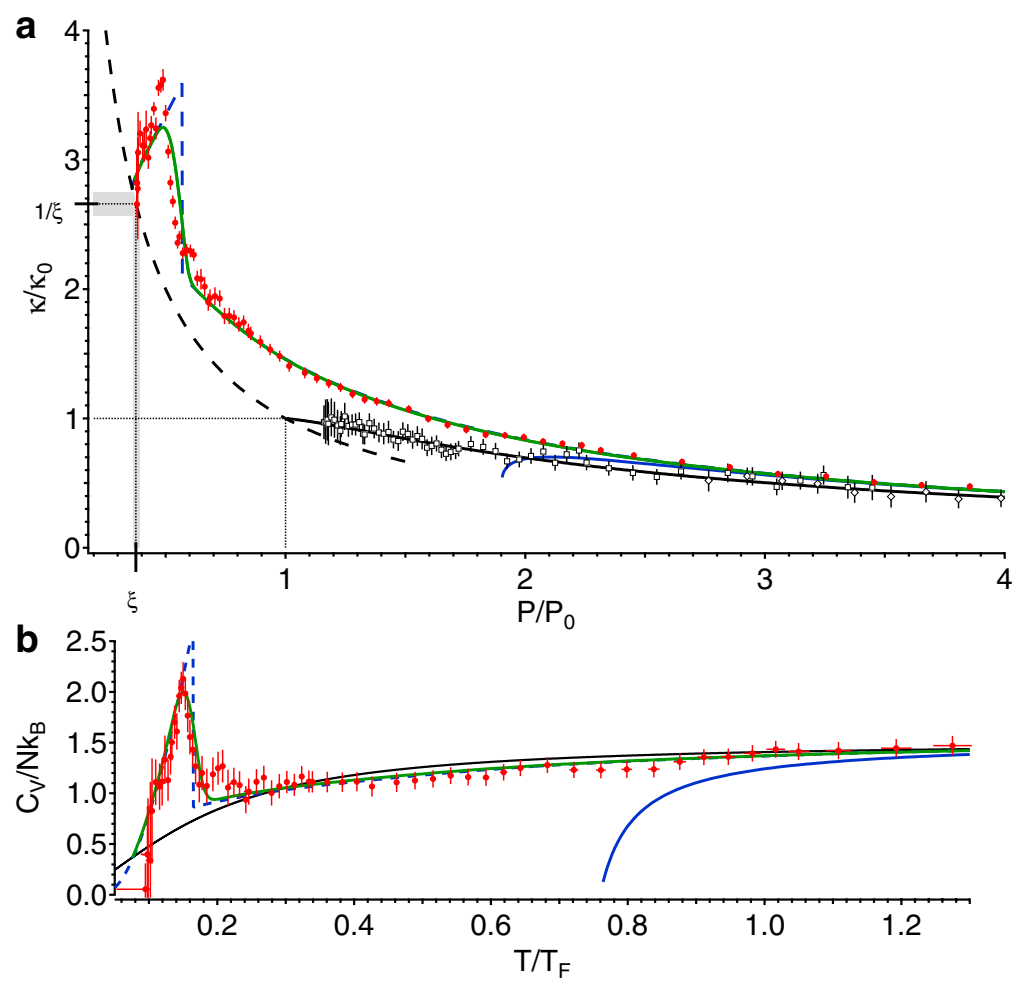

Figure 1. (a) Normalized compressibility $\tilde{\kappa}$ as a function of normalized pressure $\tilde{p}$ (red) at unitarity. Black open squares (black curve): experimental measurement (theory) for non-interacting Fermi gas. Black dashed curve: $\tilde{p}=1 / \tilde{\kappa}$ on which the $\operatorname{EoS} \tilde{\kappa}(\tilde{p})$ terminates at $T=0$. (b) Heat capacity per particle, $C_{V} / N k_{B}$, as a function of $T / T_{F}$. Red: experimental measurement at unitarity. Black curve: theory for a non-interacting gas. In both (a) and (b): the blue solid line is the third-order virial expansion; the blue dashed curve is a model incorporating diagrammatic Monte Carlo [18] above $T_{c}$, and BCS theory including phonons and pair-breaking excitations below $T_{c}$; the Green curve shows the effect of $2 \mu \mathrm{m}$ imaging resolution on the model.

parameter [20]. This implies that at $T=0, \tilde{\kappa}=1 / \xi=1 / \tilde{p}$. This yields the black dashed curve in Fig. 1 that is approached by the compressibility equation of state at low temperatures (low $\tilde{p}$ ).

From the universal function $\tilde{\kappa}(\tilde{p})$, we obtain all other thermodynamic quantities via thermodynamic relations [11]. For example, the reduced temperature $T / T_{F}$, where $T_{F}=E_{F} / k_{B}$ is the Fermi temperature, follows from a simple integral of the measured compressibility equation of state. The specific heat then follows via $\left.\frac{C_{V}}{k_{B} N} \equiv \frac{1}{k_{B} N} \frac{\partial E}{\partial T}\right|_{N, \mathcal{V}}=\frac{3}{5} \frac{\mathrm{d} \tilde{p}}{\mathrm{~d}\left(T / T_{F}\right)}=\frac{5}{2} \frac{T_{F}}{T}\left(\tilde{p}-\frac{1}{\tilde{\kappa}}\right)$, where we have used the relation $E=\frac{3}{2} P \mathcal{V}$ valid at unitarity, with $\mathcal{V}$ being the volume. The specific heat is shown in Fig. 1(b) as a function of $T / T_{F}$. For high temperatures, the heat capacity tracks that of a non-interacting gas, approaching the ideal gas value $3 / 2 N k_{B}$. A dramatic rise and fall in the heat capacity signals the superfluid transition, reminiscent of the $\lambda$-transition in superfluid ${ }^{4} \mathrm{He}[21,22]$. Jumps in the specific heat are also well-known from superconductors [23] and ${ }^{3} \mathrm{He}$ [24]. To our knowledge, this is the first time that a specific heat jump has been directly measured in an ultracold atomic gas. Previously, such jumps had only been inferred from derivatives to fit functions that implied a jump [8, 25]. We measure a critical temperature $T_{c}=0.167(13) T_{F}$. Scaled to the density of electrons in metals, this corresponds to a critical temperature for superfluidity far above room temperature.

We further obtain the chemical potential $\mu$, energy $E$, free energy $F$, and entropy $S$ of a strongly interacting Fermi gas. In Fig. 2(a), we show $\mu / E_{F}, E / E_{0}$, and $F / E_{0}$ as a function of $T / T_{F}$, where 

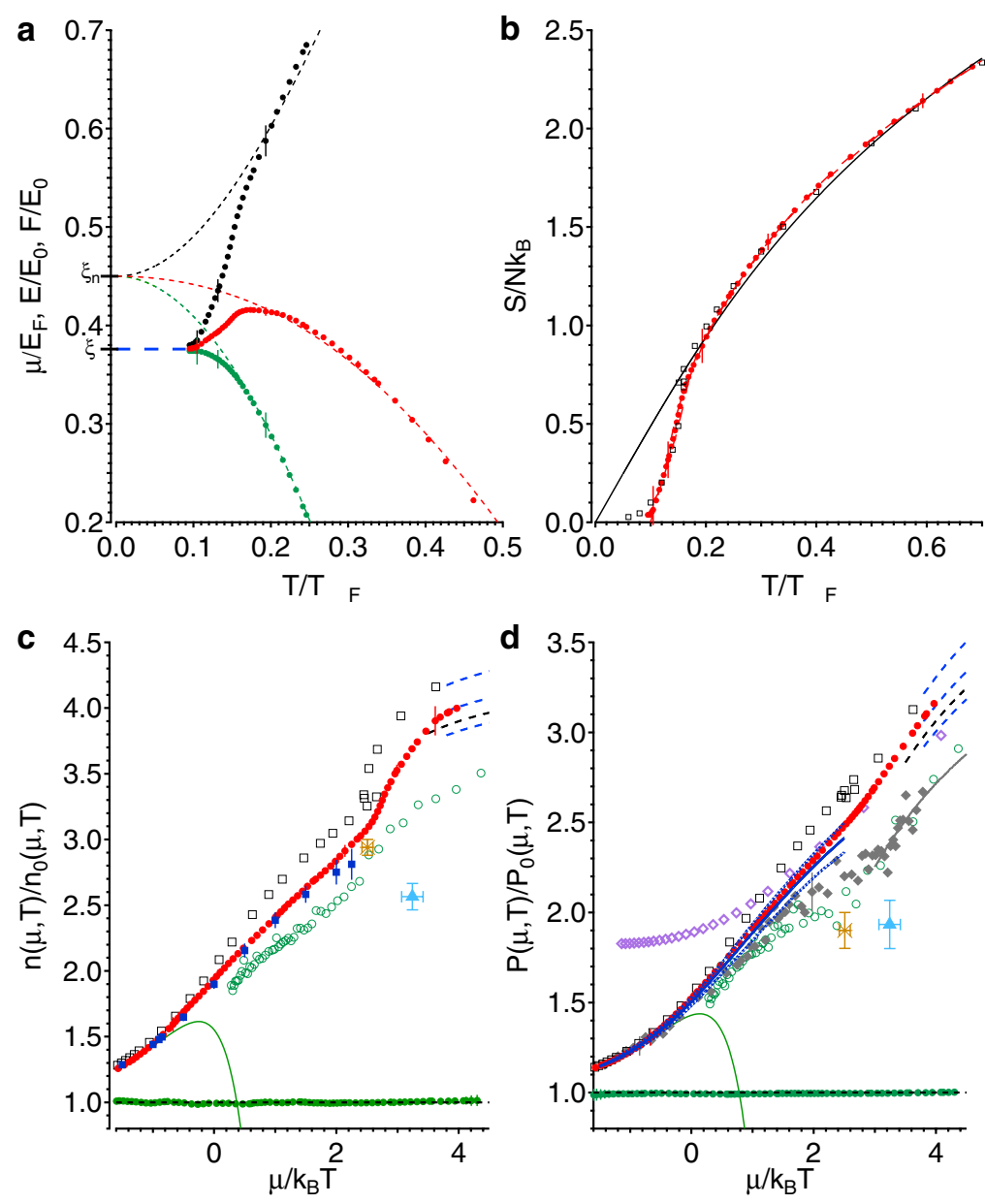

Figure 2. (a) Normalized chemical potential $\mu / E_{F}$ (red circles), energy $E / E_{0}$ (black circles), and free energy $F / E_{0}$ (green circles) as a function of $T / T_{F}$. Dashed curves: corresponding quantities for a non-interacting gas at same $T / T_{F}$ shifted by $\xi_{n}-1$, where $\xi_{n}=0.45$. (b) Entropy per particle $S / N k_{B}$ (red circles) as a function of $T / T_{F}$. Black curve: non-interacting entropy. Black open squares: $T$-matrix calculation [26]. Normalized density (c) and pressure (d) as a function of chemical potential $\mu$ and temperature $T$. Red circles: experimental measurement. Blue dashed curves: low- $T$ behavior with $\xi=0.364$ (upper), 0.376 (middle), and 0.388 (lower). Black dashed curve: low- $T$ lowerbound given by the upperbound of $\xi \leq 0.383$ [27]. Green solid circles (black fine dashed line): experimental measurement (theory) for the ideal Fermi gas. Blue solid squares: diagrammatic Monte Carlo (DMC) calculation [18] for density. Blue curve: DMC pressure, with blue dashed curves denoting the uncertainty bands. Solid green line: third-order Virial expansion [19]. Open black squares: $T$-matrix calculation [26]. Open green circles: lattice calculation [28]. Orange star and blue triangle: critical point from the Monte Carlo calculations [29] and [30], respectively. Solid diamonds: ENS experiment [9]. Purple open diamonds: Tokyo experiment [10].

$E_{0}=3 / 5 N E_{F}$ is the energy of a non-interacting gas at same density and $T=0$. The chemical potential reaches a maximum at $T_{c}$ and then drops again, consistent with the expectation of a superfluid of paired fermions. At unitarity, these quantities satisfy the following bound $E(T)>E(0)=3 / 5 \xi N E_{F}=$ $F(0)>F(T)$ for all $T$ [31]. All three quantitites approach $\xi$ as $T \rightarrow 0$. Our measurements yield the Bertsch parameter $\xi=0.376(4)$.

Fig. 2(b) shows the entropy per particle as a function of temperature. At high temperatures, the entropy tracks closely that of a non-interacting gas, and drops dramatically around the critical point, 
which we interpret as the freezing out of single-particle excitations due to pair formation. We obtain a critical entropy $S_{c}=0.73(13) N k_{B}$. The lowest entropy we achieve is less than $0.04 k_{B}$ per particle.

In Fig. 2(c) and (d), we show the density $n$ and pressure $P$ as a function of the chemical potential $\mu$ and temperature $T$, normalized by the corresponding density $n_{0}(T, \mu)$ and pressure $P_{0}(T, \mu)$ at same $\mu$ and $T$ for a non-interacting gas. This representation allows for a direct comparison with several theories for the unitary Fermi gas (see figure caption for Fig. 2).

In conclusion, we have performed a precise measurement of the equation of state of a strongly interacting Fermi gas that directly revealed the superfluid phase transition. The method developed in this work does not require an external thermometer nor a fitting procedure, and can be applied to other atomic systems such as 2D Bose or Fermi gases and fermions in optical lattices. Our measurements provide a stringent benchmark for theories of strongly interacting Fermi gases.

\section{EVOLUTION OF FERMION PAIRING FROM THREE TO TWO DIMENSIONS}

In two dimensions, two particles will form a bound state for arbitrarily weak interactions. In three dimensions, there is instead a threshold for binding, as the interaction needs to overcome the kinetic energy cost of forming the bound state. As Cooper has shown [32], two fermions on top of a filled Fermi sea can still form a bound state for arbitrarily weak attractive interactions because the Fermi sea restricts the density of available scattering states to a constant, like in 2D. In that case, the binding energy depends on the Fermi energy. How, then, does the binding energy evolve as the system is tuned continuously from three dimensions to two dimensions? How does the presence of the Fermi sea modify the binding energy? We address these questions experimentally using a gas of fermionic ${ }^{6} \mathrm{Li}$ atoms loaded into an optical lattice [12].

The binding energy is measured using radio frequency (rf) spectroscopy. Atoms are transferred from one of two initial hyperfine states into a third state by an rf pulse. In addition to response at a frequency corresponding to the atomic hyperfine splitting, we observe an asymmetric peak resulting from the dissociation of pairs (Fig. 3). The threshold of the dissociation spectrum relative to the atomic resonance gives the binding energy $E_{b}$.

Binding energies are measured for several lattice depths and scattering lengths (Fig. 4). At each scattering length, the binding energy increases with increasing lattice depth. At negative scattering lengths, where no low-energy bound state exists in 3D, non-zero binding energies are observed for sufficiently large lattice depths, in agreement with the prediction of Orso et al. [33] for two particles in a $1 \mathrm{D}$ lattice. At unitarity, the two-body binding energy is expected to approach $0.244 \hbar \omega_{z}$ for deep lattices, where $\omega_{z}$ is the harmonic approximation of the trapping frequency in a lattice well [34]. For a finite lattice depth of $20 E_{R}$, a two-body binding energy of $0.22 \hbar \omega_{z}$ is expected at unitarity [33]. We find an average binding energy of $0.232(16) \hbar \omega_{z}$ for lattice depths between 17 and $30 E_{R}$, in agreement with the two-body prediction in a deep lattice.

The agreement of the measured binding energies in $2 \mathrm{D}$ with those predicted for two isolated particles is surprising since the measurements are performed on a many-body system. This, however, is consistent with mean field theory, which predicts that in 2D, the threshold for single particle excitations from the many-body ground state is exactly given by the two-body binding energy [12, 35]. Mean field theory, however, is not expected to be accurate in the strongly-correlated regime, where the two-body binding energy is on the order of the Fermi energy. Our measurements indeed show that the measured binding energies deviate from the predicted two-body value in the center of the crossover, indicating possible beyond mean-field corrections [12].

In similar experiments at Cambridge University rf spectroscopy was applied with momentum resolution [36]. Further experiments across the dimensional crossover could elucidate the role of dimensionality in the phase diagram of strongly interacting Fermi gases and provide insight into high- $T_{c}$ superconductivity. 
EPJ Web of Conferences

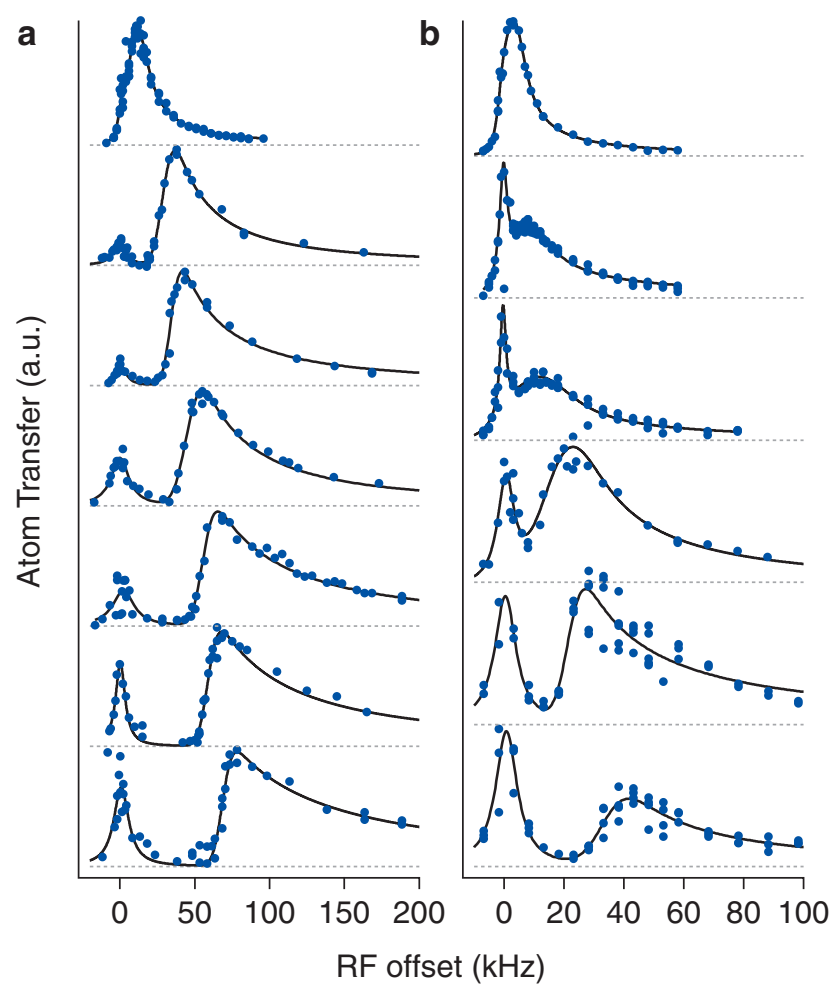

Figure 3. Evolution of fermion pairing in the $3 \mathrm{D}$ to $2 \mathrm{D}$ crossover in a one-dimensional optical lattice, observed via rf spectroscopy. (a) Spectra at the Feshbach resonance at with $d / a=-0.01(4)$. Lattice depths from top to bottom in units of $E_{R}: 1.84(3), 4.8(2), 6.1(2), 9.9(4), 12.2(4), 18.6(7)$, and 19.5(7). (b) Spectra on the BCS-side with $d / a=-1.15(2)$. Lattice depths in units of $E_{R}: 2.75(5), 4.13(7), 4.8(1), 6.0(2), 10.3(2)$, and 18.1(4).

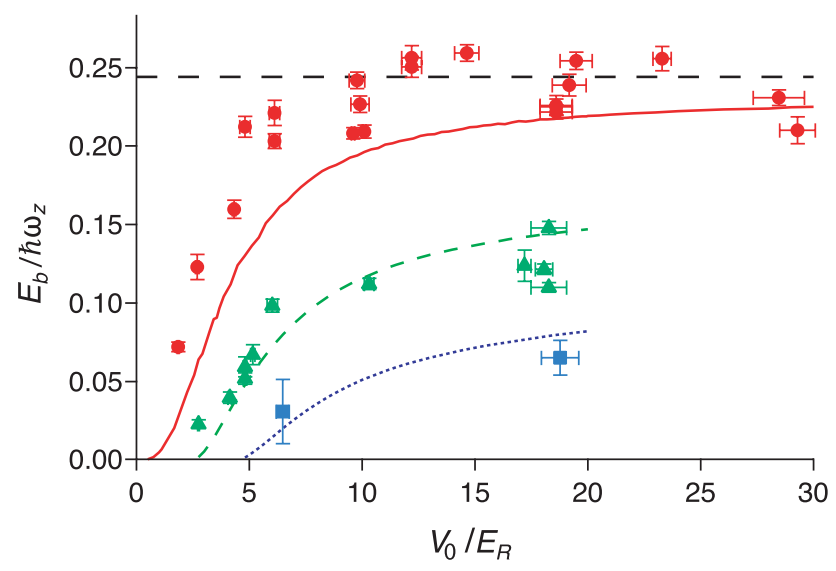

Figure 4. Binding energy $E_{b}$ versus lattice depth $V_{0}$ at several values of the $3 \mathrm{D}$ scattering length $a . E_{b}$ is normalized via the lattice frequency $\omega_{z}$. Red circles: results from spectra at $690.7(1) \mathrm{G}$ and $d / a=-0.01$ (4). Green triangles: 720.7(1) $\mathrm{G}, d / a=-1.15(2)$. Blue squares: 800.1(1) $\mathrm{G}, d / a=-2.69(1)$. Curves show predictions from Orso et al [33]. Black dashed line: harmonic approximation result for $1 / a=0$ (see Ref. [12]). 


\section{SPIN-ORBIT COUPLING IN FERMI GASES}

The superfluid phase of strongly interacting fermions is distinguished from the normal phase by spontaneous breaking of gauge symmetry. Other phases of matter, including those studied in the context of the fractional quantum Hall effect and certain spin liquids, cannot be distinguished by broken symmetries but rather by their topological order [37, 38]. The discovery of topological insulators spurred interest in topological phases that do not break time-reversal symmetry [39-42]. Such phases can be realized in lower dimensional Fermi gases with strong spin-orbit coupling in the presence of Zeeman fields. In a non-interacting 2D gas, a non-Abelian spin-orbit gauge field gives rise to topological insulators with protected edge states. Interactions lead to much richer physics; even for purely contact interactions, spin-orbit coupling gives rise to effective p-wave interactions, resulting in topological superfluids that host Majorana fermions at the interface to topologically trivial phases [43, 44].

Spin-orbit coupling, the key ingredient required for engineering these exotic phases, can be realized in atomic gases by Raman dressing of electronic states. This was demonstrated in pioneering experiments from the NIST group with Bose-Einstein condensates $[45,46]$ following theoretical proposals of the concept [47-51]. A simple geometry for obtaining spin-orbit coupling consists of two counter-propagating laser beams with a frequency difference chosen close to the energy splitting between two hyperfine states of the atom. In presence of this Raman dressing, the Hamiltonian of the system is given by

$$
\mathcal{H}=\frac{\hbar^{2} \mathbf{k}^{2}}{2 m}+\frac{\hbar^{2} Q}{2 m} k \sigma_{y}+\frac{\hbar \Omega_{R}}{2} \sigma_{z}+\frac{\delta}{2} \sigma_{y},
$$

where $k$ is the momentum of the atom, $Q$ is the momentum transferred by the Raman beams, $\Omega_{R}$ is the coupling strength and $\sigma_{i}$ measures the pseudospin along the $i$ th direction. The Doppler shift term in the Hamiltonian above can be interpreted as a momentum-dependent magnetic field coupling the spin and the momentum of the atom. In condensed matter, this type of coupling is referred to as an equal part Rashba-Dresselhaus coupling and naturally arises for example in one-dimensional quantum wires $[52,53]$. In the absence of the Zeeman field, the eigenstates of this Hamiltonian are two parabolas shifted in momentum space by $Q$ corresponding to the dispersion of each spin state. Introducing the Zeeman field opens a spin-orbit gap and the dressed states form two helicity bands with a spin texture. A BoseEinstein condensate resides in the minima of the dispersion, while a degenerate Fermi gas explores a wide range of momenta and the corresponding part of the spinful dispersion.

We have engineered spin-orbit coupling in a degenerate Fermi gas [14] of lithium by coupling the second and third lowest hyperfine states with Raman beams detuned by $3.96 \mathrm{GHz}$ to the blue of the $D_{1}$ line (also see [54] for spin-orbit coupling in a ${ }^{40} \mathrm{~K}$ Fermi gas). The difference in wavevectors of the two lattice beams is $Q=2 \pi \times(1 \mu \mathrm{m})^{-1}$, corresponding to a recoil energy $E_{R}=\hbar^{2} Q^{2} / 2 m=$ $h \times 32(1) \mathrm{kHz}$. We have focused on studying the single particle physics in this system by working at a field of $11.6 \mathrm{G}$ where the scattering length between the two spin states is very small $\left(20 a_{0}\right)$. By pulsing on the Raman beams, we observe Rabi oscillations with a frequency that depends on the momentum of the atom in the Fermi sea because of the Doppler detuning.

In order to reveal the bandstructure of the spin-orbit coupled Fermi gas, we introduce the technique of spin-injection spectroscopy which relies on energy-selective injection of fermions from reservoir states into an empty spin-orbit coupled system. We start with a spin-polarized Fermi gas occupying either the first or fourth hyperfine state and use a radio frequency (rf) pulse at a given energy to transfer fermions into the Raman-dressed states (Fig. 5(a)). Subsequently, the momentum and spin-composition of the transferred atoms is measured by spin-selective imaging after time of flight. The spinful dispersion is reconstructed from the measured spectra and the known free-particle dispersion in the reservoir states [55]. The reconstructed dispersions for different strengths of the Rabi coupling are shown in Fig. 5(b), showing the opening of the spin-orbit gap. If the chemical potential is chosen inside this gap, the gas is in a semimetallic state and the system acts as a spin diode. 

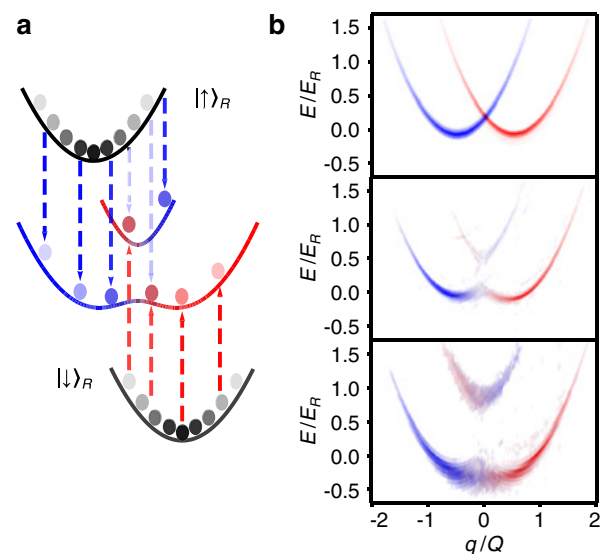

Figure 5. Spin-injection spectroscopy. (a) An rf pulse injects atoms from the reservoir states (shown in black) $|\uparrow\rangle_{R}$ and $|\downarrow\rangle_{R}$ into the spin-orbit coupled system (shown in red and blue). Injection occurs when the rf photon energy equals the energy difference between the reservoir state and the spin-orbit coupled state at quasi-momentum $q$, where $q=k_{x} \pm Q / 2$ for the two bare spin states. (b) The reconstructed spinful dispersions for $\hbar \delta=0.00(3) E_{R}$ and $\hbar \Omega_{R}=0 E_{R}, \hbar \Omega_{R}=0.43(5) E_{R}$ and $\hbar \Omega_{R}=0.9(1) E_{R}$, respectively.
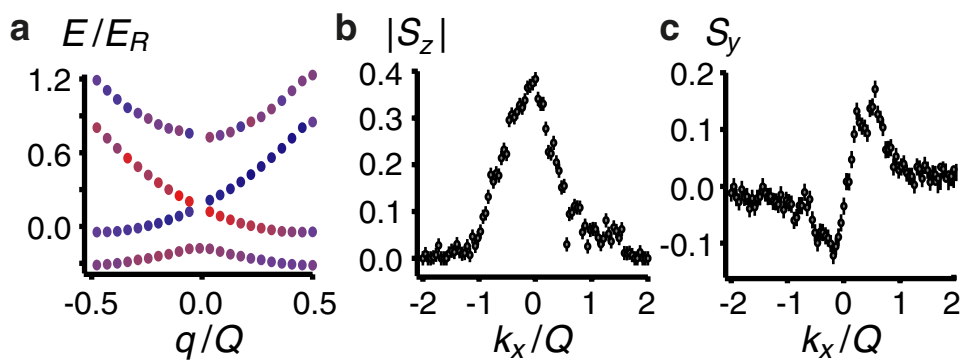

Figure 6. Energy bands of a spin-orbit coupled lattice. (a) Reconstructed band structure for $\hbar \Omega_{R}=0.93(7) E_{R}$ and $\hbar \Omega_{r f}=0.28(2) E_{R}$. Color indicates the spin texture. (b,c) Experimentally measured spin components $S_{y}$ and $\left|S_{z}\right|$ as a function of momentum $k_{x}$ for the lattice wavefunctions corresponding to the bottommost band in (a).

Spin-injection spectroscopy can be used to characterize more complicated spinful bandstructures. As an example, we have created a lattice with such a bandstructure by adding a radio frequency coupling between the bare states in the experiment described above [56]. In the previous scheme, each spin-state can only acquire or lose a momentum equal to $Q$ from the Raman process. In the presence of the rf, the atom that underwent a Raman spin-flip can be brought back into its original spin-state without changing its momentum and acquire even higher momenta through further Raman processes. The spin-orbit bandstructure described above is thus periodically reproduced in momentum space, resulting in a spinful lattice. The rf coupling opens gaps in the bandstructure, enabling it to support fully insulating states. Figure 6(a) shows the experimentally reconstructed bandstructure for the lattice and the associated spin texture for the lowest band is seen in Fig. 6(b) and (c). While the bandstructure we have studied does not possess a Zak phase, the spin-injection spectroscopy technique we have demonstrated is capable of measuring such topological invariants if they exist, although it would have to be combined with rf-induced rotations on the Bloch sphere to obtain all three spin components. 


\section{TOWARDS FERMIONIC GROUND-STATE MOLECULES WITH STRONG DIPOLAR INTERACTIONS}

Quantum gases with dipolar interactions offer another intriguing avenue for the creation of novel phases of matter. Dipolar interactions are anisotropic, long-range in character, and can be attractive or repulsive depending on the mutual orientation of the dipoles. As a consequence, dipolar matter can show nontrivial order, such as crystallization and supersolidity, support the emergence of topological phases, and enable quantum simulation and computation on the grid of a lattice structure. A promising route for the experimental realization of dipolar quantum gases is the formation of fermionic ground-state molecules with a large electric dipole moment. Pioneering experiments have succeeded in creating a gas of ${ }^{40} \mathrm{~K}^{87} \mathrm{Rb}$ ground-state molecules near quantum degeneracy [57]. However, KRb molecules are chemically unstable in their ground state, limiting their lifetimes and hampering studies of many-body physics.

Our efforts are directed towards the realization of chemically stable, fermionic ground state molecules of ${ }^{23} \mathrm{Na}^{40} \mathrm{~K}$. In addition to its chemical stability, the $\mathrm{NaK}$ molecule possesses a large induced electric dipole moment of 2.72 Debye. Therefore, fermionic ground state molecules of NaK can form a Fermi sea with strong, long-range anisotropic dipolar interactions, with an interaction energy that can exceed $30 \%$ of the Fermi energy.

Our recent studies on Bose-Fermi mixtures of ${ }^{23} \mathrm{Na}$ and ${ }^{40} \mathrm{~K}$ have revealed a quantum gas mixture with unique properties. As a first step, we have demonstrated that ${ }^{23} \mathrm{Na}$ is a highly efficient coolant for ${ }^{40} \mathrm{~K}$ [58]. When sodium is in the hyperfine state $|1,1\rangle_{\mathrm{Na}}$ and potassium in any hyperfine state of the $F=9 / 2$ manifold, three-body losses are strongly suppressed. Together with a large background scattering length ( $a_{\mathrm{bg}} \approx-700 a_{0}$, see below) and a favorable balance of forces in the optical dipole trap, this results in the predominant evaporation of sodium and efficient cooling of ${ }^{40} \mathrm{~K}$.

As a second step, we located Feshbach resonances by mapping out simultaneous atom loss of both species as a function of magnetic field [58]. We identified more than $30 s$ - and $p$-wave Feshbach resonances for low magnetic fields (below $200 \mathrm{G}$ ) in the spin channels of $|1,1\rangle_{\mathrm{Na}}$ and $\left|9 / 2, m_{F}\right\rangle_{\mathrm{K}}$ with $m_{F}=-9 / 2,-7 / 2,-5 / 2,-3 / 2$. Following a characteristic pattern, a wide $s$-wave resonance is found in each of these state combinations, the widest being located at $140 \mathrm{G}$ with an exceptional width of $30 \mathrm{G}$ between $|1,1\rangle_{\mathrm{Na}}$ and $|9 / 2,-5 / 2\rangle_{\mathrm{K}}$ atoms. We further resolved $p$-wave multiplet resonances whose multiplet structure is owed to their location at low magentic fields. The character, the position, and the widths of the resonances were validated on the basis of the asymptotic bound-state model.

The wide $s$-wave Feshbach resonances make ${ }^{23} \mathrm{Na}-{ }^{40} \mathrm{~K}$ an ideal Bose-Fermi mixture to study manybody physics, such as Bose or Fermi polarons, boson-mediated interactions between fermions or novel phases of matter in optical lattices. Furthermore, the creation of open-channel dominated and, therefore, long-lived Feshbach molecules are within reach.

Radio frequency (rf) association is a particularly clean way to form Feshbach molecules from ultracold atomic samples. It intrinsically yields accurate information on the molecular binding energy $E_{b}$ [59] and works particularly well in the vicinity of wide Feshbach resonances, where the molecular wavefunction can have a large extent and offer good overlap with two unbound atoms. We performed rf spectroscopy in the vicinity of the $|9 / 2,-3 / 2\rangle_{\mathrm{K}}$ to $|9 / 2,-5 / 2\rangle_{\mathrm{K}}$ hyperfine transition (see Fig. 7b, inset) using a balanced mixture of about 150.000 atoms of both $|1,1\rangle_{\mathrm{Na}}$ and $|9 / 2,-3 / 2\rangle_{\mathrm{K}}$. The temperature of the mixture was close to the critical temperature for Bose-Einstein condensation.

A typical rf spectrum is shown in Fig. 7a, measured on the molecular side of the wide Feshbach resonance between $|1,1\rangle_{\mathrm{Na}}$ and $|9 / 2,-5 / 2\rangle_{\mathrm{K}}$. It has two characteristic features: an atomic peak near the unperturbed hyperfine transition, and a molecular peak revealing ${ }^{40} \mathrm{~K}$ atoms that have been associated into $\mathrm{NaK}$ molecules. We typically create $20 \times 10^{3}$ molecules, corresponding to a conversion efficiency of about $15 \%$ of the initial ${ }^{40} \mathrm{~K}$ atoms. The distance between the atomic and the molecular peak yields the binding energy $E_{b}$. 


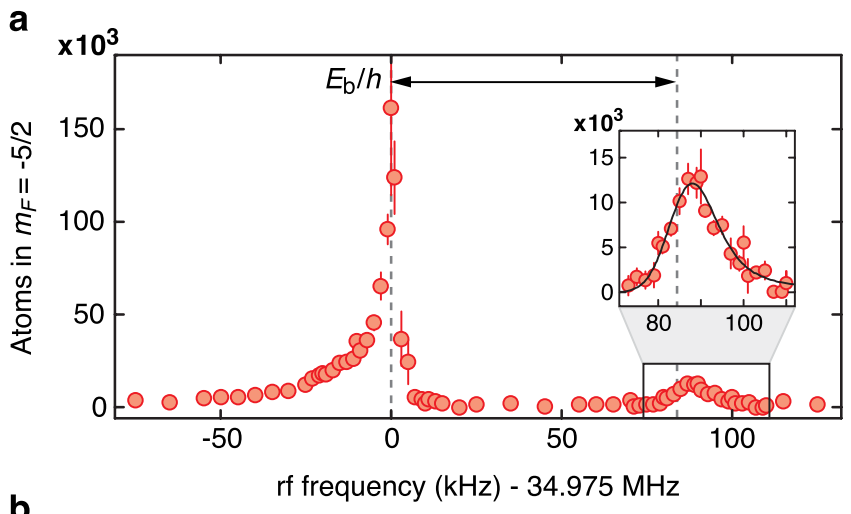

b

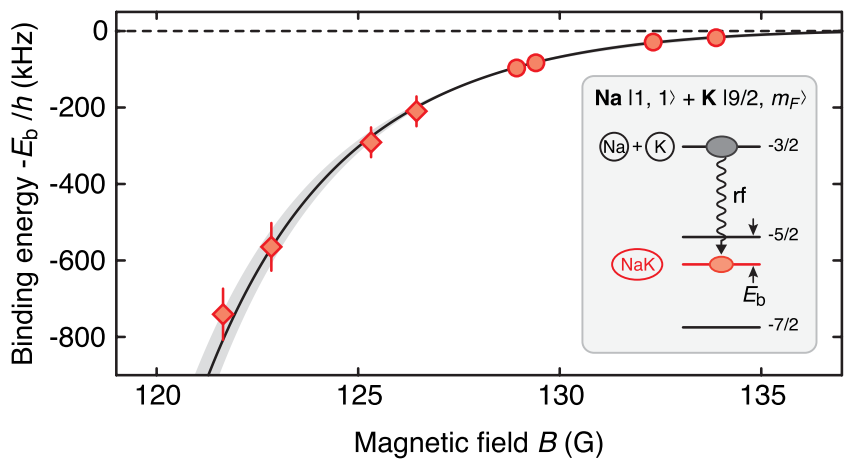

Figure 7. (a) Association of fermionic Feshbach molecules. Radio frequency spectroscopy near the $|9 / 2,-3 / 2\rangle_{\mathrm{K}}$ to $|9 / 2,-5 / 2\rangle_{\mathrm{K}}$ hyperfine transition reveals two distinct features: Free ${ }^{40} \mathrm{~K}$ atoms repulsively interacting with the ${ }^{23} \mathrm{Na}$ bosonic bath near zero rf offset, and, near $85 \mathrm{kHz}$ rf offset, associated Feshbach molecules. A fit to the molecular association spectrum (inset), which takes into account the Franck-Condon factor and the probability density for pair formation, yields a binding energy of $E_{b}=h \times 84(6) \mathrm{kHz}$. The magnetic field was $129.4 \mathrm{G}$. (b) Binding energies of NaK Feshbach molecules at the wide Feshbach resonance between $|1,1\rangle_{\mathrm{Na}}$ and $|9 / 2,-5 / 2\rangle_{\mathrm{K}}$. Binding energies $E_{b}$ below $200 \mathrm{kHz}$ (circles) are obtained by direct detection of molecules (see (a)); larger binding energies are measured via simultaneous atom loss in $|1,1\rangle_{\mathrm{Na}}$ and $|9 / 2,-5 / 2\rangle_{\mathrm{K}}$.

The shape of the molecular peak is determined by the thermal distributions of $\mathrm{Na}$ and $\mathrm{K}$, the FranckCondon factor between bound and unbound Na-K pairs, as well as the finite experimental resolution. A fit including these effects (see inset of Fig. 7(a)) allows us to obtain an accurate value for the binding energy at a given magnetic field. The molecular binding energy as a function of magnetic field is shown in Fig. 7(b). The quadratic dependence of the binding energy reflects the open-channel character over a wide magnetic field range, where the binding energy follows $E_{b} \propto 1 /(a-\bar{a})^{2}$ with the mean scattering length $\bar{a}=51 a_{0}$ and $a(B) \approx a_{\mathrm{bg}}\left[1+\Delta B /\left(B-B_{0}\right)\right]$. Based on the asymptotic bound-state model we determine a large and negative background scattering length $a_{\mathrm{bg}}=-690_{-130}^{+90} a_{0}$, a resonance position of $B_{0}=139.7_{-1.4}^{+2.1} \mathrm{G}$, and a width of $\Delta B=29(2) \mathrm{G}$.

Studying the time-of-flight expansion of NaK Feshbach molecules via direct absorption imaging, we determined an effective temperature of $500 \mathrm{nK}$, corresponding to a degeneracy factor of $T / T_{\mathrm{F}, \mathrm{mol}} \approx 1.7$. Additionally, we observed a remarkably long molecular lifetime of about $8 \mathrm{~ms}$ (at $E_{b}=h \times 32 \mathrm{kHz}$ ) even in the presence of unassociated, remaining $\mathrm{Na}$ and $\mathrm{K}$ atoms. Utilizing the species-selectivity of the depth of our optical dipole trap $(\lambda=1064 \mathrm{~nm})$, we were able to remove bosonic Na atoms from the sample and observed strongly enhanced molecular lifetimes of more than $100 \mathrm{~ms}$ in the vicinity 
of the Feshbach resonance. The corresponding loss rate due to collisions of molecules with leftover $|9 / 2,-3 / 2\rangle_{\mathrm{K}}$ atoms is $\beta=8(2) \times 10^{-12} \mathrm{~cm}^{3} / \mathrm{s}$. This is three times lower than the lowest loss rate measured for $\mathrm{KRb}$ molecules.

In our studies we have created near-degenerate, long-lived fermionic Feshbach molecules of NaK, a molecule that is chemically stable in its absolute ground state. From our measurement of the molecular binding energies, we deduce that the Feshbach molecules are open-channel dominated over a large magnetic field range, which goes along with significant singlet character. The observed conditions are ideal to perform a two-photon STIRAP transfer into the rovibrational ground state. In view of an induced dipole moment of 2.72 Debye and chemical stability, NaK constitutes an ideal candidate to create a strongly dipolar Fermi gas with dominating long-range anisotropic interactions - a novel quantum resource with yet unforeseen possibilities in quantum simulation, quantum computation and quantum chemistry.

\section{CONCLUSION AND OUTLOOK}

These proceedings summarize our recent experiments on Fermi gases, with the unifying theme of understanding and creating strong interactions. Our method for determining the thermodynamics of ultracold gases can be applied to a variety of novel systems under current investigation, such as the lower dimensional Fermi gases (section 3) and spin-orbit coupled Fermi gases (section 4). Generally, in the quest to go beyond $s$-wave pairing, towards novel states of fermionic matter that might have topological properties, a variety of possibilities open up, from using laser beams to modify the dispersion relation in spin-orbit coupling (section 4) to harnessing dipolar interactions between fermionic molecules (section 5). Each method comes with their own challenges, but also their own advantages, and the more interesting systems are found, the larger the probability that we find new, exotic, but stable and robust states of matter.

\section{References}

[1] M. Inguscio, W. Ketterle, C. Salomon, eds., Ultracold Fermi Gases, Proceedings of the International School of Physics “Enrico Fermi”, Course CLXIV, Varenna, 20-30 June 2006 (IOS Press, Amsterdam, 2008).

[2] W. Ketterle, M. Zwierlein, Rivista del Nuovo Cimento 31, 247 (2008).

[3] S. Giorgini, L. P. Pitaevskii, S. Stringari, Rev. Mod. Phys. 80, 1215 (2008).

[4] M.W. Zwierlein, J.R. Abo-Shaeer, A. Schirotzek, C.H. Schunck, W. etterle, Nature 435, 1047 (2005).

[5] J. Kinast, A. Turlapov, J.E. Thomas, Q. Chen, J. Stajic, K. Levin, Science 307, 1296 (2005).

[6] J.E. Thomas, J. Kinast, A. Turlapov, Phys. Rev. Lett. 95, 120402 (2005).

[7] J.T. Stewart, J.P. Gaebler, C.A. Regal, D.S. Jin, Phys. Rev. Lett. 97, 220406 (2006).

[8] L. Luo, J. Thomas, J. Low Temp. Phys. 154, 1 (2009).

[9] S. Nascimbène, N. Navon, K. Jiang, F. Chevy, C. Salomon, Nature 463, 1057 (2010).

[10] M. Horikoshi, S. Nakajima, M. Ueda, T. Mukaiyama, Science 327, 442 (2010).

[11] M.J.H. Ku, A.T. Sommer, L.W. Cheuk, M.W. Zwierlein, Science 335, 563 (2012).

[12] A.T. Sommer, L.W. Cheuk, M.J.H. Ku, W.S. Bakr, M.W. Zwierlein, Physical Review Letters 108, 045302 (2012).

[13] A.Y. Kitaev, Annals of Physics 303, 2 (2003).

[14] L.W. Cheuk, A.T. Sommer, Z. Hadzibabic, T. Yefsah, W.S. Bakr, M.W. Zwierlein, Phys. Rev. Lett. 109, 095302 (2012).

[15] T.L. Ho, Phys. Rev. Lett. 92, 090402 (2004). 
[16] F. Pistolesi, G.C. Strinati, Phys. Rev. B 53, 15168 (1996).

[17] Y. Shin, M. Zwierlein, C. Schunck, A. Schirotzek, W. Ketterle, Phys. Rev. Lett. 97, 030401 (2006).

[18] K. Van Houcke, F. Werner, E. Kozik, N. Prokof'ev, B. Svistunov, M.J.H. Ku, A.T. Sommer, L.W. Cheuk, A. Schirotzek, M.W. Zwierlein, Nature Phys. 8, 366 (2012).

[19] X.J. Liu, H. Hu, P.D. Drummond, Phys. Rev. Lett. 102, 160401 (2009).

[20] G. Bertsch, Proceedings of the Tenth International Conference on Recent Progress in Many-Body Theories, in Recent progress in many-body theories, edited by R. Bishop, K.A. Gernoth, N. R. Walet, Y. Xian (World Scientific, Seattle, 2000).

[21] W. H. Keesom, A. P. Keesom, Physica 2, 557 (1935).

[22] J. A. Lipa, J. A. Nissen, D. A. Stricker, D. R. Swanson, T. C. P. Chui, Phys. Rev. B 68, 174518 (2003).

[23] M. Tinkham, Introduction to Superconductivity, 2nd edn. (Dover, Mineola, New York, 2004).

[24] D. Vollhardt, P. Wölfle, The Superfluid Phases of Helium 3 (Taylor \& Francis, London, 1990).

[25] J. Ensher, D. Jin, M. Matthews, C. Wieman, E. Cornell, Phys. Rev. Lett. 77, 4984 (1996).

[26] R. Haussmann, W. Rantner, S. Cerrito, W. Zwerger, Phys. Rev. A 75, 023610 (2007).

[27] M.M. Forbes, S. Gandolfi, A. Gezerlis, Phys. Rev. Lett. 106, 235303 (2011).

[28] A. Bulgac, J.E. Drut, P. Magierski, Phys. Rev. Lett. 96, 090404 (2006).

[29] O. Goulko, M. Wingate, Phys. Rev. A 82, 053621 (2010).

[30] E. Burovski, N. Prokof'ev, B. Svistunov, M. Troyer, Phys. Rev. Lett. 96, 160402 (2006).

[31] Y. Castin, F. Werner, in The BCS-BEC Crossover and the Unitary Fermi Gas, edited by W. Zwerger (Springer-Verlag, Berlin, 2012), chap. 5.

[32] L. N. Cooper, Physical Review 104, 1189 (1956).

[33] G. Orso, L.P. Pitaevskii, S. Stringari, M. Wouters, Physical Review Letters 95 (2005).

[34] I. Bloch, J. Dalibard, W. Zwerger, Rev. Mod. Phys. 80, 885 (2008).

[35] M. Randeria, J. M. Duan, L.Y. Shieh, Physical Review Lettres 62, 981 (1989).

[36] M. Feld, B. Fröhlich, E. Vogt, M. Koschorreck, M. Köhl, Nature 480, 75 (2011).

[37] D. J. Thouless, M. Kohmoto, M.P. Nightingale, M. den Nijs, Phys. Rev. Lett. 49, 405 (1982).

[38] X. G. Wen, Advances in Physics 44, 405 (1995).

[39] C. L. Kane, E. J. Mele, Phys. Rev. Lett. 95, 226801 (2005).

[40] B. A. Bernevig, S. C. Zhang, Phys. Rev. Lett. 96, 106802 (2006).

[41] M. Z. Hasan, C. L. Kane, Rev. Mod. Phys. 82, 3045 (2010).

[42] X. L. Qi, S. C. Zhang, Rev. Mod. Phys. 83, 1057 (2011).

[43] R. M. Lutchyn, J. D. Sau, S. Das Sarma, Phys. Rev. Lett. 105, 077001 (2010).

[44] V. Mourik, K. Zuo, S. M. Frolov, S. R. Plissard, E. P. A. M. Bakkers, L. P. Kouwenhoven, Science 336, 1003 (2012).

[45] Y. J. Lin, R. L. Compton, K. Jiménez-García, J. V. Porto, I. B. Spielman, Nature 462, 628 (2009).

[46] Y. J. Lin, K. Jiménez-García, I.B. Spielman, Nature 471, 83 (2011).

[47] J. Higbie, D. M. Stamper-Kurn, Phys. Rev. Lett. 88, 090401 (2002).

[48] S. L. Zhu, H. Fu, C. J. Wu, S. C. Zhang, L. M. Duan, Phys. Rev. Lett. 97, 240401 (2006).

[49] T. D. Stanescu, B. Anderson, V. Galitski, Phys. Rev. A 78, 023616 (2008).

[50] X. J. Liu, M.F. Borunda, X. Liu, J. Sinova, Phys. Rev. Lett. 102, 046402 (2009).

[51] J. Dalibard, F. Gerbier, G. Juzeliūnas, P. Öhberg, Rev. Mod. Phys. 83, 1523 (2011).

[52] C. H. L. Quay, T. L. Hughes, J. A. Sulpizio, L. N. Pfeiffer, K.W. Baldwin, K. W. West, D. Goldhaber-Gordon, R. de Picciotto, Nat. Phys. 6, 336 (2010).

[53] S. Nadj-Perge, V. S. Pribiag, J. W. G. van den Berg, K. Zuo, S. R. Plissard, E. P. A. M. Bakkers, S. M. Frolov, L. P. Kouwenhoven, Phys. Rev. Lett. 108, 166801 (2012).

[54] P. Wang, Z.Q. Yu, Z. Fu, J. Miao, L. Huang, S. Chai, H. Zhai, J. Zhang, Phys. Rev. Lett. 109, 095301 (2012).

[55] J. T. Stewart, J. P. Gaebler, D. S. Jin, Nature 454, 744 (2008). 
[56] K. Jiménez-García, L.J. LeBlanc, R. A. Williams, M. C. Beeler, A. R. Perry, I. B. Spielman, Phys. Rev. Lett. 108, 225303 (2012).

[57] K.K. Ni, S. Ospelkaus, M. H. G. de Miranda, A. Pe'er, B. Neyenhuis, J. J. Zirbel, S. Kotochigova, P. S. Julienne, D. S. Jin, J. Ye, Science 322, 231 (2008).

[58] J. W. Park, C. H. Wu, I. Santiago, T. G. Tiecke, S. Will, P. Ahmadi, M. W. Zwierlein, Phys. Rev. A 85, 051602 (2012).

[59] C. H. Wu, J. W. Park, P. Ahmadi, S. Will, M. W. Zwierlein, Phys. Rev. Lett. 109, 085301 (2012). 